\title{
DOWN SYNDROME DETECTION USING MODIFIED ANT COLONY OPTIMIZATION ALGORITHM
}

\author{
Vincy Devi V K \\ Research Scholar, Department of Computer Science \\ Bharathiar University, Coimabatore, India \\ vincydevi15@gmail.com \\ Dr. Rajesh R \\ Associate Professor, Department of Computer Science, \\ CHRIST (Deemed to be University), Bangalore, India \\ ryanrajesh@hotmail.com
}

\begin{abstract}
Nowadays, the systems related to healthcare are restructured with innovative skills to offer humans more intellectual and proficient healthcare facilities. Various intelligent healthcare systems are exhibited with the help of machine learning and artificial intelligent tools to offer intellectual and expert services. In human body genetic codes are stored in the genes. All of our inherited traits are associated with these genes and are grouped as structures generally called chromosomes. In typical cases, each cell consists of 23 pairs of chromosomes, out of which each parent contributes half. But if a person has a partial or full copy of chromosome 21, the situation is called Down syndrome. It results in intellectual disability, reading impairment, developmental delay, and other medical abnormalities. This paper introduces an intelligent prediction and classification system for healthcare, feature selection based on density with Ant Colony Optimization (ACO) algorithm for Down syndrome (DS).
\end{abstract}

Keywords: Down Syndrome;Ant Colony Optimization (ACO); Backpropogation Neural Network (BPNN); Fiducial Points

\section{Introduction}

Nature is an immense source of encouragement for resolving hard and complex problems in computer science since it exhibits an extremely diverse, dynamic, robust, complex, and attractive phenomenon. It always finds the optimal solution to solve its problem maintaining perfect balance among its components. This is the thrust behind bio-inspired computing. Nature-inspired algorithms are meta-heuristics that mimic nature for solving optimization problems opening a new era in computation. For the past decades, numerous research efforts have been concentrated in this particular area. Still being young and the results being very amazing, broadens the scope and viability of Bio-Inspired Algorithms exploring new areas of application and more opportunities in computing. This work proposes a broad overview of biologically inspired optimization algorithm - Ant Colony based Optimization. The proposed intelligent system excludes inappropriate or redundant features before the structure of ACO based classifier. The proposed method has three phases namely pre-processing, Feature Selection, and classification [1]. Comparing the proposed algorithm with existing methods, the presented intelligent system outperformed the other methodologies with a significant improvisation in classification accuracy using fewer features.

Thus, early detection and screening of this disability are the best styles for Down syndrome prevention. In this work, Down syndrome detection utilizes a collection of facial expression images and classifies these images based on the age. A compact geometric descriptor is employed for extracting the facial features from the image set. Ant Colony based Optimization algorithm is applied to gather the required data sets and for the classification. The extracted information is then normalized and used to train the Neural Network using the Backpropagation algorithm [2]. This paper proposes an algorithm to detect the chance for DS through the analysis of fetus facial characters. Localization of facial points is done and the intercanthal distance is calculated. Using these calculations, the proposed algorithm detects the chance for DS. For recognizing DS, it is important to consider the intercanthal distance - the space between the inside recesses of the eyes. People with DS often present addition in the intercanthal distance called telecanthus. The occurrence of small palpebral fissures, which is the distance between the lateral and medial canthus of the optics is another common feature. People with DS commonly indicate the symptom of a flattened nose and the presence of a small mouth.In this proposed method, the facial landmarks are detected and then a geometric descriptor is built using it. Saraydemir et.al [3] proposed a Gabor wavelet transform technique for the detection of DS and the technique possesses high accuracy. Burçin and Vasif developed a Local Binary Pattern (LBP) based approach for the DS diagnosis. Here they used a template matching 
strategy. Still, these methods take some sort of manual pre-processing for the success of the diagnosis process. Boehringer et.al, [4] studied the characteristic facial patterns using Gabor wavelet features for ten syndromes, excluding Down syndrome. Further studies [5] give a classification for 14

\section{Related work}

Medical imaging plays an important role in the detection and identification of diseases in humans, and medi calimage processing has a wide variety of applications, so researchers have made substantial contributions for many years. Various image processing tools and algorithms were used to recognize Down syndrome by extracting the facial features and classifying the images into different classes. This section shows the merits and limitation of the existing models used in recommendation system

Kruszka P., et al. [18] proposed a new method based on the observation results and digital facial recognition technology in people with Down syndrome from various populations. On 65 people from 13 countries, photos and clinical data were collected. This research shows the broad range of findings in Down syndrome across various regional communities and highlights the accuracy and potential of digital facial recognition technology in Down syndrome diagnosis around the world. This research used images of facial and limb findings to subjectively classify DS, as well as objectively using facial recognition technology and collectively organizing clinical exam findings from the medical literature. The aim of this study was to provide clinicians with a baseline reference for diagnosing Down syndrome in a variety of populations, as an earlier diagnosis allows for more effective preventive measures, early detection of complications including congenital heart disease, and genetic therapy and recurrence risk discussions with parents.

This research has a range of possible limitations. One of the most difficult aspects of the research was researching people of various ages. Many studies concentrate on a particular age group, such as newborns, because facial features change with age. Furthermore, since much of the data in this and other studies was subjective and dependent on examiner judgment, they have used digital facial recognition technology to illustrate this by extracting texture features from inner facial land marks, A Support Vector Machine classifier is trained using leave-one-out strategy cross-validation. The number of features that maximized classification efficiency was chosen as the optimal number for each classification. The proposed system could recognize the Down syndrome with $94.3 \%$ accuracy.

Burçin K. and Vasif N. V [19] introduced a new method for recognizing Down syndrome by using the local binary pattern (LBP) approach for feature extraction which is a very efficient feature descriptor. The methods of Euclidean distance and Changed Manhattan distance were used for classification. As a result, they were able to develop an effective method for detecting Down syndrome. Different facial expressions, races, light conditions, and facial wears such as glasses, hair, and so on complicate the issue. They attempted to recognize Down syndrome from images of faces in the study. They looked at face samples and used a descriptor called local binary pattern (LBP) to extract facial features. To make the system more robust, they used two classifiers; Euclidean distance and Changed Manhattan distance methods. The results of the two classifiers differ, and one of them is correct. The Euclidean distance result for a Down sample is down, while the Changed Manhattan distance result is not down, and when the results are combined based on the differences, the system result is down, as expected. Despite many variations such as ethnicity, skin tone, facial wear, age, and pose, they built an improved system with $95.3 \%$ accuracy.

Erogul O., et al. [20] described a new method in which image processing algorithms were applied to images of Down syndrome patients' faces in order to create a pre-diagnostic tool. Children between the ages of 5 and 6 were the subjects of the data sets analyzed in this analysis. 18 images of children were examined in each of the typical syndrome classes. For all images, the critical points on faces are calculated using the elastic face bunch graph process. For both preparation and classification, ten feature vectors were added to an artificial neural network. As a consequence, using a neural network, Down syndrome can be prediagnosed with a $68.7 \%$ precision.

\section{Proposed Approach}

The proposed work operates in three stages: preprocessing, FS, and classification. The preprocessing stage is the primary process since the database may contain redundant and noisy data. By examining the data, different processes take place such as data cleaning, filling missing values, removing excessive data because the missing values and excessive data degrade the performance. In this work, a few features are selected with the help of the feature selection process. The purpose of using a wrapper method is the selection of the best feature subset by repeatedly generating a set of features till the best subset is obtained. To register the obtained feature vector, the ACO based classification approach is employed for classifying the data. This combination of density-based feature selection and ACO algorithm allows the user to predict and diagnose the health using their medical data. The proposed ACO model will attain high classification performance with few features and achieve optimal performance measurements [6][7]. 


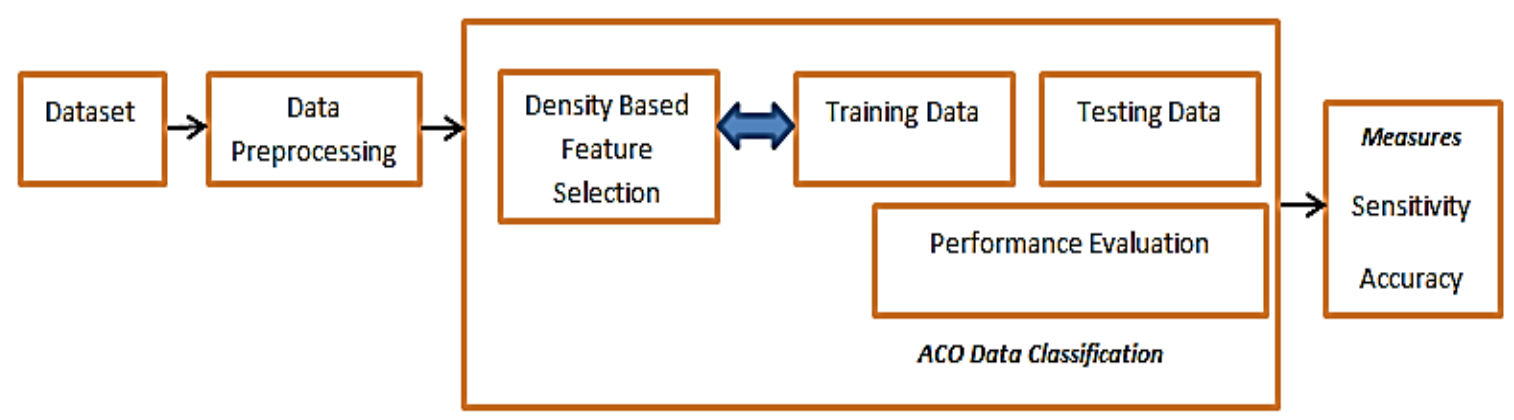

Figure 1: Block Diagram of ACO

For the selection of optimal features, the suggested algorithm uses the succeeding processes. Once the preprocessing of the dataset is accomplished, the next step is to organize the input data into groups. Here, densitybased feature selection is used which chooses a group of features in every single iteration. A subdivision of the optimal features from the raw dataset is measured as the most important feature for the classification procedure. This process is a heuristic methodology used to estimate the merits of features. The main assumption of considering a feature is good when every class has less overlap with the remaining classes. For the classification task, the ACO algorithm is used for the extraction of classification rules, using the behavior of ant colonies and data mining techniques [8].

\subsection{Backpropagation Neural Network (BPNN)}

A neural network [9] is defined as a collection of associated input-output elements such that each connection has a weight linked with it. It is applied to make predictive models from big databases and they mimic the human neural system. For training feature sets extracted from the classifier, multi-layer BPNNs are used. Commonly, a multi-layer network consists of three layers of nerve cells. They are the input layer, a hidden layer, and output layer. The connections in BPNN are such that, each node in the input layer is connected to the nodes of the hidden layer. Similarly, hidden layer nodes are related to the output layer. There is no direct link between the nodes in an individual layer and with every connection; there should be an associated weighting factor [10]. At the time of training, the weights are modified using the BP algorithm and the process is called learning. In this study, the supervised learning method is adopted. The ANN training parameters are presented in Table 1.

Table 1: ANN training parameters

\begin{tabular}{ccccc}
\hline No. of Inputs & No. of outputs & No. of Hidden Layer & No. of Hidden Neurons & $\begin{array}{c}\text { No. of } \\
\text { iterations }\end{array}$ \\
\hline 7 & 1 & 1 & 8 & 500 \\
\hline Leaming Rate & Momentum Rate & Activation Function & Training Method & Error \\
\hline 0.05 & 0.5 & Sigmoid & Backpropagation & 0.001 \\
\hline
\end{tabular}

The difference between target and actual response is termed as the system error. During the training process, this error is backpropagated to the preceding layer, i.e. the hidden layer. The error is then calculated for each element in this layer $\mathrm{N}$. In the same manner, error at each node of a previously hidden layer that is $\mathrm{N}-1$ is also determined. By using this method to reduce the error to the expected level.

\subsection{Ant Colony Optimization (ACO)}

ACO is applied in various fields of optimization and is introduced by Dorigo and Gambardella in 1997 [11]. This algorithm is exhibited on the action of an ant colony. It is a probabilistic procedure useful in problems that compact with verdict improved paths through graphs established on the performance of ants pursuing a path between their colony and a source of food. An artificial ant plays a significant role in tracing optimal solutions by moving through a parameter space on behalf of all possible solutions. Natural ants lay down pheromones guiding the others to resources while exploring their environment. The artificial ants similarly record their position and the quality of their solutions, so that in later simulation iterations more ants locate better solutions [12-14]. 


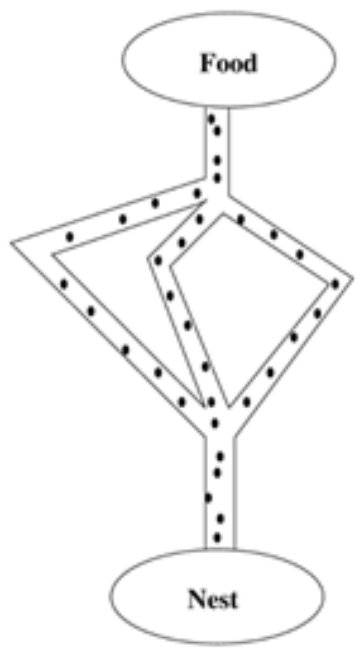

(a)

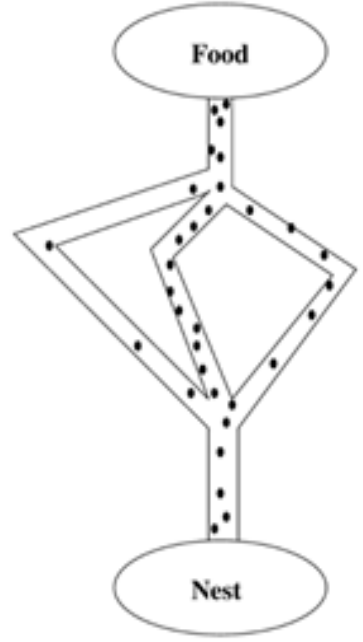

(b)

Figure 2: ACO algorithm. (a) The initial status. (b) The shortest path found with pheromone.

The ACO is a conventional swarm intelligent technique for interpreting combinatorial optimization problems. The main inspiration behind the ACO algorithm is the concept of 'stigmergy' in nature. Stigmergy is a method of self-organization and it refers to the modification of the environment by biological organisms to communicate with each other. Indirect communication takes place when an individual configures the environment and at a later time, others respond to that modified environment. In an ant colony, all the ants are regularly searching for food around the nest aimlessly. 'Pheromone' is used by ants for local communication. These hormonal chemicals are generated and emitted by an organism to give information to the other members of their species. Ant marks their traveling path with pheromones when they reach a food source. The quality and quantity of the food source are highly proportional to the amount of emitted pheromones [15].

Ant deposits strong and concentrated pheromone if they found a better and more source of food. Other ants recognize the existence of the pheromone and follow it to reach the food source. Ants carry the collected food to their nest and mark their path to the next. This is how the ants find the shortest path between the nest and a food source. Vaporization takes place when ants deposit pheromones. The pheromone updating period is inversely proportional to the length of the path between the nest and source of food. It shows that the shortest path becomes more concentrated as more ants get attracted. A finite set of available solutions are used to create artificial ants. Each ant takes values from this finite set. An ant can select the entire unvisited node available from the current position in its every iteration [16][17].

An ant ' $\mathrm{k}$ ' at node ' $\mathrm{r}$ ' will choose the destination node ' $\mathrm{s}$ ' at a later stage with a probability as given in Equation (1)

$$
P_{k(r, s)}=\frac{\tau_{r, s}^{\alpha} \eta_{r, s}^{\beta}}{\sum \tau_{r, s}^{\alpha} \eta_{r, s}^{\beta}}
$$

where $\tau_{r, s}$ shows the amount of pheromone of r, s edge, $\alpha$-defined the impact of pheromone, $\eta_{r, s}$ indicates the desirability of $r, s$ edge, and $\beta$-defines the impact of the desirability.

\subsection{Proposed Algorithm}

(1) Initialize the process by setting the parameters like pheromone value, heuristic information, number of ants, and stopping function.

(2) Construct the solution for each ant and then evaluate the constructed solutions. Repeat the process until exit criteria are met. If the criterion is not met then update the pheromone value using the ACO.

(3) Calculate the probability.

(4) Choose the system with the highest priority and calculate the time.

(5) Calculate the quantity of deposition of pheromones.

(6) Adapt the pheromone value. 
(7) On the occasion that the exit criterion is satisfied; update the initial weights of BP.

(8) Train the BP network and obtain MSE. Again the process checks for the exit criterion.

(9) If the condition fails repeat from step 3. Otherwise, save the results for MSE and Regression.

\section{Results and Discussion}

Fiducial points are points that are used as points of reference or measure; hence determining the fiducial points is the fundamental step to recognize a face. Some of the main fiducial points are the eyes, lip edges, nose, chin, etc. In this study, some images from the Dartmouth Database of Children's Faces are selected, inclusive of males and female images that were showing very distinct facial expressions. The images were actually captured spontaneously from different backgrounds, angles, and distances, showing varying head poses, facial expressions, and occlusions. As the next step, the image is tested and verified and then compared to the solution with other prominent classifiers. The proposed methodology mainly composed of four stages: (i) facial detection, (ii) features extraction, (iii) features reduction, and (iv) classification. It can be remarked that from the study that as the number of iterations increased, there is a diminution in the error value. BPNN is used to train the classified parameters and MATLAB is the program utilized for training and testing. Figure 3 shows the performance of the ACO algorithm.

Following, we compare the anticipated response of BP- ACO with other existing methods. The given system has improved performance than the later model. The accuracy of the proposed method is better than the other methods. This is the main purpose to use BP for parameter optimization and as a weak classifier; this system is hybrid with ACO technology. Enhanced prediction accurateness has provided by a stronger classifier.

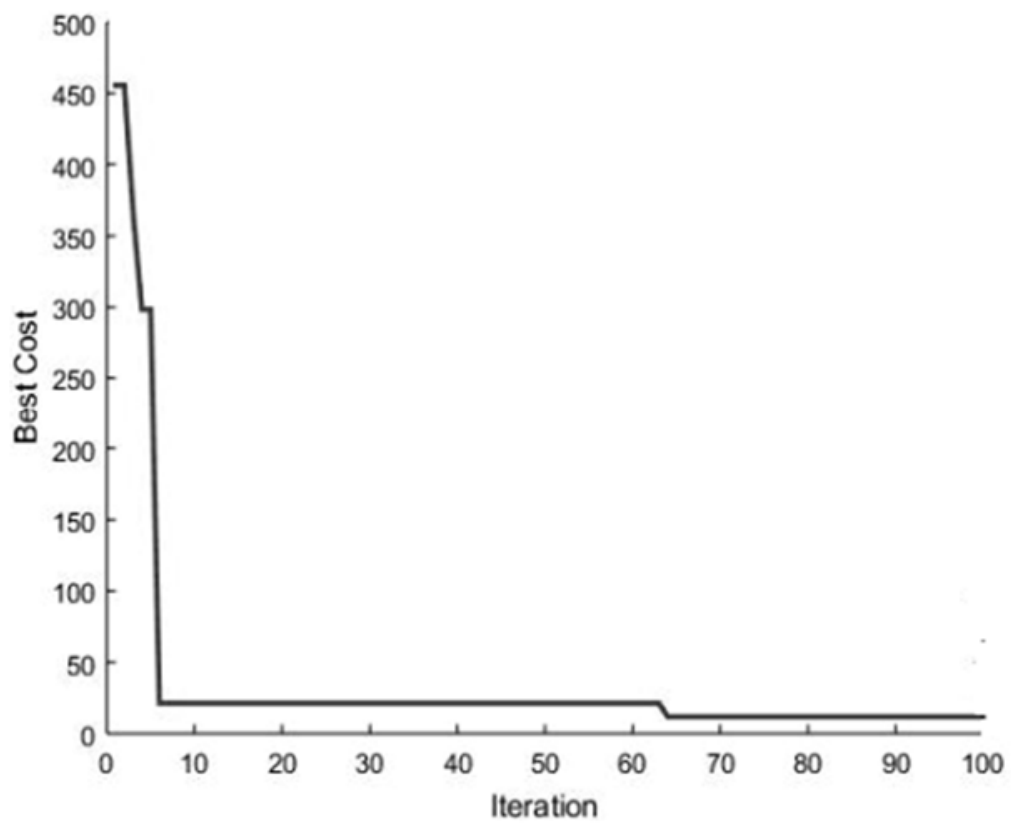

Figure 3: Best cost vs the number of iterations

Fig. 4 shows the network architecture of the presented ANN system. The performance of the network is analyzed in provisions of MSE and Epochs. The graph shows the trends of training, validation and test data in terms of MSE and Epochs. From the graph, it is clear that the MSE shrinks with an increase in the figure of epochs for all trained, validation and test data. The accuracy of the system is $97.81 \%$ on the basis of performance parameters. Table II shows the comparison of the proposed system with other works.

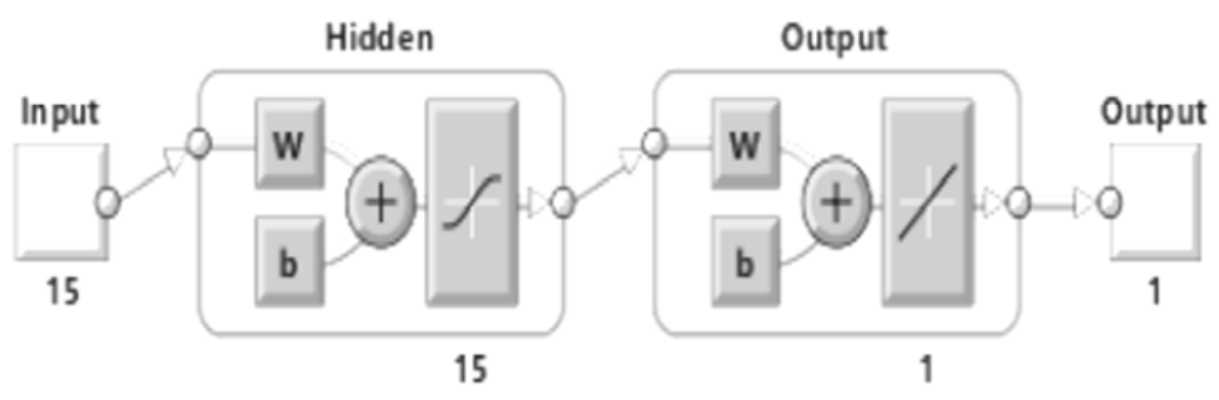

Figure 4: Network Architecture of the presented system 
Table 2: Accuracy Comparison

\begin{tabular}{lcccc}
\hline \multicolumn{1}{c}{ Access } & Scheme & Number of images & Accuracy (\%) \\
\hline Kruszka et.al & {$[18]$} & SVM & 65 & 94.30 \\
Burcin and Vasif [19] & LBPs & 107 & 95.30 \\
Erogul et.al & {$[20]$} & EBGM & 86 & 68.70 \\
Proposed Model & MACO & 82 & 97.81 \\
\end{tabular}

\section{Conclusion}

An ant colony optimization-based technique is used for the early detection of Down syndrome is proposed. There is no proper treatment for Down syndrome; so its early detection is indispensable and necessary. The proposed technique is based on facial identification and localization of facial points of the fetus. By computing the length between these points, the proposed algorithm predicts the chance for Down syndrome. Facial identification is performed with the help of ACO and neural network training is performed with the help of MATLAB. Further, it is possible to increase the accuracy by increasing the number of training iterations and selecting more sensitive facial localization points. The accuracy of the proposed system is $97.81 \%$.

\section{References}

[1] M. Dorigo and T. Stutzle "Ant Colony Optimization: Overview and Recent Advances", Handbook of Metaheuristics, vol. 272, 2018, pp. 311-351.

[2] Lv, Chen \& Xing, Yang \& Zhang, Junzhi \& Na, Xiaoxiang \& Li, Yutong \& Liu, Teng \& Cao, and Dongpu \& Wang, Fei-Yue. "Levenberg-Marquardt Backpropagation Training of Multilayer Neural Networks for State Estimation of a Safety-Critical CyberPhysical System," IEEE Transactions on Industrial Informatics vol 14 (8), 2017, pp. 1-10.

[3] Ş. Saraydemir, et al., "Down Syndrome Diagnosis Based on Gabor Wavelet Transform," Journal of Medical Systems, vol. 36, pp. 3205$3213,2012$.

[4] S. Boehringer, et al., "Automated syndrome detection in a set of clinical facial photographs," American Journal of Medical Genetics Part A, vol. 155, pp. 2161-2169, 2011.

[5] T. A. Anjit and S. Rishidas, "Identification of nasal bone for the early detection of Down syndrome using Back Propagation Neural Network," 2011 International Conference on Communications and Signal Processing, Calicut, 2011, pp. 136-140, doi: 10.1109/ICCSP.2011.5739286.

[6] Kuan Foong, Wai \& Maier, Holger \& Simpson, Angus, "Power plant maintenance scheduling using ant colony optimization: An improved formulation," Eng. Optim. vol. 40(4), 2008, pp. 309-329.

[7] Aaron C.Zecchin, Angus R.Simpson, Holger R.Maier, Michael Leonard, Andrew J.Roberts and Matthew J.Berrisford, "The Application of two ant colony optimization algorithms to water distribution system optimization" Mathematical and Computer Modeling, vol. 44, 2006, pp. 451-468.

[8] C. Solnon, I. Alaya and K. Ghédira, “Ant colony optimization for multi-objective optimization problems,"- International Conference on Tools with Artificial Intelligence- Proceedings, vol. 1, 2007, pp. 450-457.

[9] Vladislav Skorpil and Jiri Stastny, "Neural Networks and Back Propagation Algorithm," Electronics. Bulg. Sozopol, Bulgaria, 2006, pp. 173-176

[10] Tiwari, Sheela \& Naresh, Ram \& Jha, and Rahul, "Comparative Study of Backpropagation Algorithms In Neural Network-Based Identification Of Power System," International Journal of Computer Science and Information Technology, vol. 5, 2013, pp. 93-107.

[11] Dorigo, Marco, and Luca Maria Gambardella. "Ant colonies for the travelling salesman problem." Biosystems, vol. 43(2) ,1997, pp. 7381.

[12] Korošec, Peter \& Silc, Jurij \& Filipic, and Bogdan “The differential ant-stigmergy algorithm", ” Information Sciences vol 192, 2010, pp. 82-97.

[13] Joy, V. M., \& Krishnakumar, S., Optimal design of adaptive power scheduling using modified ant colony optimization algorithm. International Journal of Electrical \& Computer Engineering, vol. 10(1), 2020, pp. 738-745.

[14] Francis Heylighen, "Stigmergy as a universal coordination mechanism I: Definition and components," Cognitive Systems Research, vol. 38 (2016), pp. 4-13.

[15] Serra, M \& Venini, P, "On some applications of ant colony optimization metaheuristic to plane truss optimization," Structural and Multidisciplinary Optimization, vol. 32(6), 2006, pp. 499-506.

[16] Baterina, Anna Veronica, and Carlos Oppus. "Image edge detection using ant colony optimization." Wseas transactions on signal processing, vol. 6(2), 2010, pp. 58-67.

[17] Vijo M Joy, S. Krishnakumar, " A Modified Ant Colony Optimization Algorithm for Power Scheduling," International Journal of Innovative Technology and Exploring Engineering, Vol. 8 (11),2019, pp. 1083-1088.

[18] Kruszka P., et al. , "Down syndrome in diverse populations," Am. J. Med. Genet. Part A 173 (1), 42-53 (2017). 10.1002/ajmg. a. v173. 1 [PubMed] [CrossRef] [Google Scholar]

[19] Burçin K. and Vasif N. V., "Down syndrome recognition using local binary patterns and statistical evaluation of the system," Expert Syst. Appl. vol. 38, no. 7,pp. 8690-8695 (2011).10.1016/j.eswa.2011.01.076.

[20] Erogul O., et al. , "Recognition of Down syndromes using image analysis," in 14th National Biomedical Engineering Meeting, pp. 14, IEEE; (2009). 10.1109/BIYOMUT.2009.5130322 [CrossRef] [Google Scholar] 


\section{Authors Profile}

Mrs. Vincy Devi V. K is working as Assistant Professor in the department of Computer
Applications, Sree Narayana Gurukulam College of Engineering, Kadayinuppu, Emakulam.
She has 12 years of teaching experience She is doing her research in Medical Image
processing. Her area of interest include Computer Networking, Cloud Computing and
Bigdata Analysis.

